\title{
Post-Trial Considerations for an Early Phase Optogenetic Trial in the Human Brain
}

\author{
Michael White', Roger G Whittaker ${ }^{1,2}$ \\ 'Translational and Clinical Research Institute, Newcastle University, Newcastle upon Tyne, NE2 4HH, UK; ${ }^{2}$ Department of Clinical Neurophysiology, \\ Royal Victoria Hospital, Newcastle upon Tyne, NEI 4LP, UK
}

Correspondence: Michael White, Translational and Clinical Research Institute, Newcastle University, Framlington Place, Newcastle upon Tyne, NE2 4HH, UK, Tel +44 0 I 9 I 208 328I, Email Michael.white2@ncl.ac.uk

\begin{abstract}
For many clinical trials, the issue of post-trial access to research treatments is straightforward. Sponsors offer a range of follow-on studies, compassionate use programs or expanded access programs to allow participants to continue accessing beneficial experimental treatments. But there are times when this is not always the case and participants are required to stop beneficial treatments and return to standard care. Guidance states that post-trial access should be made available for "those participants who still need an intervention identified as beneficial". This broad statement has allowed sponsors to make their own interpretation of when an intervention is still "needed" and when it is "beneficial". As a result, there have been a number of situations where participants of clinical trials have been left afterwards with feelings of abandonment. Participants involved in studies with long-term, invasive treatments can be seen as being particularly vulnerable. Optogenetic technology has the potential to offer hope to people with neurological conditions, especially people who may not respond to current approved treatments. Optogenetics typically involves two components: a gene therapy medicinal product (GTMP) that induces long-term expression of light-reactive proteins within cells, and an active implantable device to stimulate the light-sensitised cells. Neither works without the other, hence for long-term patient benefit, both must remain active and may therefore require maintenance or replacement. With the potential life-long consequences of both components and the difficulty of accessing the brain, there is a need to reconsider post-trial guidelines and whether they are suitable to support early phase optogenetic trial participants. This paper considers the ethical and regulatory requirements in place for post-trial access and care in relation to optogenetic treatments of neurological conditions. We propose that a new perspective with wider responsibilities for sponsors is required when it comes to these types of novel therapies.
\end{abstract}

Keywords: optogenetics, post-trial care, clinical trial, neuroscience, trial ethics

\section{Plain Language Summary}

Clear guidelines exist on how participants should be treated at the end of drug trials. Organisations such as the World Medical Association recommend that benefitting trial participants should continue to receive access for as long as stocks last or until the drug is licenced. However, this is open to exploitation by companies where benefits may vary widely between study participants or when other treatments are available. Losing access to beneficial experimental treatments can leave participants with feelings of loss and abandonment.

New optogenetic therapies for use in the brain pose a number of additional challenges for post-trial care. These treatments involve the lifelong expression of light-reactive proteins within brain cells and placing an implant within the brain to control abnormal brain activity. The long-term nature of the therapy means it comes with long-term risks, many of which are currently unknown. Furthermore, there are limited options for removing the transfected neurons or the brain implant. Since neither works without the other, failure of either risks leaving participants in a worse situation than if they had never taken part in the trial.

This paper proposes changes in standard post-trial arrangements that will safeguard participants. Recommendations include pushing companies to use technology that is unlikely to become obsolete in the future and to cost and plan for long-term participant support from the early planning stage. This will ensure that participants are treated respectfully and kept at the centre of research, while providing long-term safety and efficacy data to benefit future participants. 


\section{Introduction}

Stakeholders in medical research trials have a responsibility to provide continued access to investigational products. The World Medical Association states in the 2013 Helsinki Declaration that "sponsors, researchers and host country governments should make provisions for post-trial access for all participants who still need an intervention identified as beneficial in the trial". ${ }^{1}$ Similar guidance for the provision of continued access to interventions demonstrating significant benefit has been released by the Council for International Organizations of Medical Sciences in collaboration with the World Health Organization. ${ }^{2}$ The need to plan for the follow up of trial participants involving advanced therapy medicinal products and active implantable medicinal devices is also detailed in regulatory guidance. ${ }^{3,4}$ However, the guidance provided by these organisations is short on detail and open to interpretation by sponsors. For example, the definition of significant benefit from a treatment is unclear and the guidance is vague on whether or not benefit from a treatment should be determined on an individual or group basis. While guidance has been published to help clarify posttrial responsibilities for medicines, ${ }^{5}$ the same is not the case for medical devices.

The standard practice among sponsors for post-trial access to investigational medicines is that it is provided only when the following conditions are met: ${ }^{6}$

- The condition being treated is serious or life-threatening and/or long term.

- The participant is benefitting and withdrawal of treatment will adversely affect health or wellbeing.

- There is no local availability of alternative treatment.

- There is sufficient efficacy and safety data and a positive risk/benefit profile for the experimental treatment.

When post-trial access is approved, it is often done so in the form of follow-on studies, compassionate use programs or expanded access programs. These will run until a product becomes licensed at which point responsibility for provision passes to the local health-care system. However, the lack of guidance outside of medicinal trials allows sponsors to minimise the provision of post-trial care. This is of particular concern as treatments become more invasive. This is exemplified with the emerging technology of optogenetics which offers the potential for highly specific, closed-loop control of cellular activity for neurological conditions such as epilepsy and depression.

Optogenetics involves the introduction of light-reactive proteins called opsins into cells via the use of viral vectors. Expression of these opsins within neuronal cells can allow clinicians to alter activity through the use of optical stimulation, delivered by a neural implant. Opsins come in a variety of different forms with excitatory and inhibitory opsins capable of stimulating or inhibiting neuronal activity. Opsins also range in their mode of operation and response speed providing the possibility of millisecond precision control over neural networks. The use of promoter genes can also allow expression within specific targeted neuronal subtypes. ${ }^{7}$ This opens up the possibility of treating a range of conditions including cardiac arrhythmia, sight and hearing loss, urinary bladder disease as well as neurological conditions such as migraine, epilepsy and Alzheimer's. ${ }^{8-11}$

As optogenetic technology continues to develop, many research institutes and companies are working towards early phase clinical trials. To date, there have been four clinical trials involving optogenetics, all of which are for the treatment of Retinitis Pigmentosa. ${ }^{12-15}$ Initial limited data released from the Allergan and Nanoscope Therapeutics studies suggest that an optogenetic gene therapy treatment is well tolerated. ${ }^{16,17}$ However, it must be noted that Allergan have so far only published data on one patient, and the data from Nanoscope Therapeutics are yet to be published in a peer-reviewed journal. Progress in the use of optogenetics for visual conditions is aided by the accessible nature of the eye and lack of requirement for an internalised light source. The need to develop an implant capable of safely introducing light within the body means that optogenetic trials for other conditions may take another 5-10 years. While implantable optrode technology is widely used in rodents and non-human primates, it is yet to be trialled in humans. With advances being made in size and biocompatibility for neurotechnology, the future for clinical grade optrode technology appears bright. ${ }^{18}$

Trial sponsors spend considerable time considering the aims and objectives for a trial as well as the procedures and investigations necessary to meet them. But alongside trial protocols, long-term plans also need to be drawn up. These may include follow-on studies or open label registries and are planned in the expectation of success. Yet study success may not necessarily match with individual success. Long-term plans must take into account all eventualities including 
situations where a therapy may fail efficacy goals at the group level, but where some participants individually benefit. This is not an uncommon finding in trials of neuromodulation; for example, vagus nerve stimulators typically produce beneficial effects in only a subset of patients, but for those that do respond the benefits can be significant. ${ }^{19}$

The need for provision of post-trial access to investigational therapies has a number of arguments including both medical and ethical perspectives. Ethical arguments propose that post-trial plans must take into account respect for the physical and emotional investment that participants bring to a trial. An early phase trial of an optogenetic therapy within the brain will be high-risk and intrusive. The risks from a GTMP will vary depending upon the type of viral vector selected. A vector such as lentivirus that integrates the opsin gene into the host DNA has the potential to cause insertional mutagenesis that could result in tumour formation. ${ }^{20}$ There may also be an immune response by the body towards a vector or towards the opsins expressed by cells. The presence of opsins may also prove toxic to a cell leading to cellular damage or cell death. Due to the novel nature of optogenetics, the long-term expression of opsins within human cells has not been proven meaning there is a risk that levels of opsin expression may fall over time affecting efficacy and requiring repeat injections. This is something that is not straightforward for neurological conditions.

The use of an implantable device introduces its own risks with any foreign entity to the body potentially causing damage to tissue or provoking an immune response. ${ }^{21}$ If tissue damage occurs during the insertion of a device, then glial tissue may form around the implant affecting efficacy and the overall success of any optogenetic treatment. Any invasive surgical procedure would also have its own risks such as infection. This is something that can have serious consequences, particularly within the brain.

Therefore, there is an ethical duty to support ongoing post-trial access to participants due to the high level of burden that they would take on in a trial. These include allowing the lifelong expression of non-human proteins within the brain, allowing implantation of the neural device, undergoing invasive surgeries and attending extensive appointments. As a result, if at the end of a trial a participant is seeing benefit from the experimental treatment, then a sponsor can recognise their contribution to research by providing ongoing access to care.

A second ethical argument for providing continued access to treatment is that of non-maleficence. This is the belief that patients should not be intentionally harmed or left in a worse position after a trial than if they had not participated in the first place. With investigational pharmaceuticals, this is less likely to be an issue as a product's active substance will be cleared from the body over time. So if access to treatment is removed, then participants will return to the same situation as they were before the trial. However, this cannot be the case for optogenetics. A GTMP, normally a viral vector, is required for an optogenetic therapy to induce long-term expression of opsins within targeted cells. While the vector itself may be short lived, being developed to be incapable of undergoing replication, the genes delivered are not. Combined with the long life of neuronal cells, expression of the medicinal product can be expected to be lifelong. Due to the nature of these light reactive proteins, it can be expected that neuronal activity will remain unaffected as long as there is no exposure to light. However, the integration of the transgene and production of opsins may be associated with longterm risks such as mutagenesis and cell toxicity. ${ }^{22}$ The presence of an implant within the brain also has potential longterm consequences for patients with ongoing risks of infection, chemical leaching and bowstringing. ${ }^{23}$ These risks can be acceptable when the transgene is combined with a working implanted device to produce modulation of network activity due to the potential for benefit. However, once either component fails, falls into disrepair or is removed, then, without the possibility of benefit, any level of risk becomes unacceptable.

Gene therapy regulations do require a long-term post-trial monitoring plan for patient safety even in the event of product failure. $^{24,25}$ This will be particularly important due to the current lack of any long-term safety data from opsin expression in the human brain. This requirement to undertake long-term safety monitoring of the GTMP may help to persuade sponsors to include continued access to a therapy.

At the end of some neural implant trials, the implant is switched off but not explanted by the sponsor. ${ }^{26}$ While this removes the risks involved with surgery, it means that the participant must continue to live with an implant within the body and the risks inherent with that. For example, if an implant is not MRI compatible, then a patient may be disadvantaged by an inability of medical staff to identify and treat future medical conditions. This is particularly relevant in the case of an optogenetic trial where one of the theoretical risks associated with the gene therapy is the development of a brain tumour, for which MRI scanning is an important investigation. Removing the implant involves a risk of 
damage from the surgical procedure itself, or from the production of scar tissue that may further damage tissue and restrict patient movement ("bowstringing"). It should also be noted that as neural technology advances, not all implants may be capable of being removed. Implant technology is becoming increasingly diverse with variation in shapes, sizes and materials from DBS leads and Utah arrays to fine neural grains and threads. Neural threads in particular are designed to integrate as fully as possible within the brain in order to minimise any immune system response. However, the delicate nature of the implants along with the expected cellular response over time is likely to make removal of neural threads close to impossible without causing significant damage to tissue. Rigid electrode arrays and leads may be easier to remove but cause greater cellular damage upon insertion. With some level of damage expected from the necessary surgical procedures, and the possibility that neural stimulation itself may affect long-term network behaviour ${ }^{27}$ it means that participants from an optogenetic trial will likely end up worse off to some degree without active therapy. The nonmaleficence argument therefore supports the provision of continued access should any benefit be seen during the trial.

A key argument put forward against the provision of post-trial access to experimental treatments is the availability of alternative approved treatments. Due to the highly intrusive nature of optogenetics, an optogenetic-based treatment is likely to be a final line of treatment where patients have tried and failed to respond to standard treatments. For example, in the case of epilepsy, which is a strong candidate for the use of optogenetics, first-line treatment is medication of which a range must have been tried before clinicians will look to more intrusive surgical options. The expectation might be for the inclusion criteria of an early phase trial to target these non-responders. Therefore, at the end of a trial, there would be a lack of suitable therapeutic alternatives to be transferred to as they had already been previously tried. Of course, this may not always be the case. For example, some focal epilepsy patients may be eligible for resective surgery that could be delayed to trial the slightly less intrusive optogenetic therapy. In this situation, a participant could be provided with the clinically approved resective surgery at the end of a trial. For other conditions that could be targeted by optogenetics such as depression and Parkinson's disease, it may also be possible that alternative therapies are available. However, the use of a gene therapy will typically exclude participants from the use of other or future gene therapies, further limiting the availability of alternative treatments and increasing the requirement to provide ongoing access.

The presence of alternative treatment options reduces but does not altogether remove the argument for providing continued access to treatment, especially if the experimental treatment is giving substantial benefit and the alternative treatment has its own risks. Transferring a patient from a treatment showing benefit to one clinically approved but untried by a patient might lead to a deterioration in a condition, placing participants at risk of harm from a relapse of symptoms that were otherwise being controlled. If a change of treatment comes with a risk of reduced control of a condition, then again there is a strong ethical justification against withdrawing the beneficial, yet experimental, treatment.

Any ethical arguments must be considered as part of a risk/benefit assessment for a new therapy. While an experimental treatment may provide benefit to a participant, it also comes with risks. For an early phase optogenetic study, these risks are likely to be largely unknown and take several years to appear such as the long-term immune response, the life-span of batteries and effects of transduction. As patients receive treatment, data will be collected that will continuously alter the risk/benefit ratio. If post-trial access to treatment is provided, then important long-term safety and efficacy data can continue to be collected. Should any long-term safety concerns be identified, it may become possible that the risks from a therapy outweigh the benefits. If this point is reached, then the safety data may well justify withdrawal of treatment on the grounds of patient wellbeing.

Most reasons for stopping product development are due to safety or efficacy reasons. But the free market nature of healthcare means that in some circumstances a developer may decide to stop a trial for business reasons. This can leave participants having a beneficial treatment withdrawn due to perceived commercial risk to a company as opposed to safety risks to themselves. MRCT guidance for medicines supports this position for sponsors, also stating that sponsors are under no obligation to continue manufacturing products discontinued for business or strategic reasons. ${ }^{5}$ In the case of a drug trial, this means that participants might only be able to get post-trial access to a treatment until stocks run out, however long that might be. Optogenetic therapy patients will already have the GTMP and implant and so long-term issues will relate to accessing replacement batteries and leads as well as software updates and expertise to collect, analyse and adjust device settings. ${ }^{28}$ If manufacturers make the decision to halt the development and production of parts or software updates, participants will be left with the possibility that their implant becomes obsolete and incompatible with future technology. Outdated, specialist 
technology can also leave patients confined to seeing small groups of specialists for treatment with local establishments illequipped to deal with the technology. This can place participants at an increased risk in medical emergency situations. At the start of the BROADEN study in the United States, which investigated the use of DBS for depression, the sponsor Abbott agreed to pay the cost of device removal at the end of the study or the supply of rechargeable batteries for those wishing to keep their device. ${ }^{29}$ All other costs related to the ongoing use would be the responsibility of the patient but having medical insurance might not guarantee cover. Research at one US centre found that around two-thirds of off-label Deep Brain Stimulation procedures were not reimbursed despite being preapproved. ${ }^{30}$ With current neurotechnology trials, these implications, along with the possibility that participants may become financially liable for future treatment, leave many with no option other than to undergo surgery to remove an implant at the termination of a trial as the sponsor's business decisions alter the risk/benefit ratio for patients. ${ }^{26}$

It is not unreasonable to accept that should a technology be unsuccessful, then companies cannot be expected to continue with expensive research and development for a handful of responders. This is especially the case if those funds can arguably be put to better work elsewhere. However, companies can plan ahead to put in place support for any responders by ensuring a surplus of parts at the start of a trial and by using industry standard procedures and connections. This will increase the likelihood of an implant being compatible with other future technologies. For smaller organisations who may be dependent upon trial success to survive, this forward planning is especially important. In the event a company should fold for financial reasons, then there may be some hope of ongoing access for those responders. A lack of planning may leave all participants with no option but to stop treatment and manage the resulting clinical and psychological repercussions on their own.

While much is made of ensuring ongoing support for those patients benefitting from a treatment, it is important to acknowledge that not all may benefit from an optogenetic treatment. And for non-responders an end of treatment may not necessarily be straightforward. Patients may wish to have their implant removed rather than continue living with an inactive device within their body, particularly if they have negative associations with it. However, this is a procedure that sponsors are under no obligation to provide and may not agree to cover on the basis that the implant can simply be switched off. ${ }^{31}$ In the US, insurance companies are also under no obligation to cover costs and will rarely do so unless removal is shown to be medically necessary. So with companies unwilling to pay, the cost of removal falls to the participant who has already undergone a lot without promise of benefit. ${ }^{32}$ And yet upon removal, companies will often be able to claim ownership of an implant, gaining access to the device and valuable data on how a device responds to being placed within the brain environment.

It is also important for sponsors to look at the wider support made available to trial participants when they come to the end of a trial and transfer back to standard clinics. ${ }^{33}$ For while a research study may end with a last patient last visit, the fallout for those participants will carry on as they deal with changes in lifestyle, disease management and emotions. Guidance documentation concentrates on post-trial access, ignoring the wider post-trial care needed for participants. This care will often fall to local health-care systems with occasional support from researchers and research institutes. As it is seen as outside of the protocol, and not necessary to answer the research question, sponsors will often ignore this important aspect of care. But with sponsors and researchers collecting data that will potentially lead to large benefits for them, there is little ethical justification around the release of participants at the end of a trial. And for participants who put themselves forward for high-risk procedures without promise of benefit, there is a growing acceptance that research teams have a wider responsibility than just the tasks detailed within a protocol. ${ }^{34}$

The complexity of this is highlighted in the participant experiences of a three-month trial involving insulin pumps that needed returning at the end due to a lack of funds. For patients who were assigned to the experimental arm of the trial, returning the pumps at the end brought a range of emotions. Some reported anxiety about the loss of new found freedoms gained during the trial, of becoming deskilled in routine diabetes management and of the concern over reinstating old, restrictive lifestyle regimes. ${ }^{35}$ For participants taking part in research involving fully invasive technology lasting years, these experiences are likely to be magnified. This was played out for study participants who were forced to give up a neural implant that provided warnings for seizures when the sponsor failed to obtain further funding, As one participant stated, "It was like taking away that part of myself that made me complete". ${ }^{26}$ 
Therefore, supporting a patient beyond the end of a trial is an important part of pastoral care, especially if a trial lasts a number of years and the end of a trial results in a change in circumstances. Yet it is at this point that the blurred relationships between sponsor, researcher and participant are revealed. When joining a research study, an informed consent form is signed by the participant and researcher. Despite being written by the sponsor, they are not a signatory. As such, there is no formal documented relationship between the sponsor and a trial participant. ${ }^{36}$ It is also the researcher that the participant will see regularly and so a duty of care above and beyond merely undertaking research procedures will often be created. This is particularly the case for high-risk interventional studies where patients may have run out of treatment options. ${ }^{37}$ This can be expected to be the case with an early phase optogenetic trial, where the long study duration is likely to create strong relationships between participants and researchers. Therefore, researchers will have been given a moral duty to ensure participants are not seen as merely data points and that their best interests are prioritised from a point of view of engagement, compassion and gratitude. This can be seen as particularly important when the use of a GTMP may make someone ineligible for other treatments, potentially limiting their future treatment options. As the point of contact between participants and sponsors and the lack of contractual obligation between those two parties, the burden falls upon researchers to ensure sponsors put the necessary requirements in place to ensure patient wellbeing.

There has been much debate about the impact that a neural implant might have on a person's sense of identity. ${ }^{38-40}$ With cell specificity and the limited spread of light within the brain, optogenetics can ensure that only a very small part of the brain is altered. The risk of an optogenetic therapy impacting on global brain function is likely to be minimal. However, the impact that a treatment may have on a person's sense of self may be independent of any therapeutic modification of cellular activity. Instead it may rely more upon the changes a treatment places on someone's relationship with their condition. For example, a patient who has not come to terms with a condition may struggle with an implant that provides a regular reminder of that condition. This may be the case even if that implant is shown to be efficacious. Conversely, a patient may form a strong bond with a device if it is felt to link with improved circumstances even if experimental data shows a treatment to have negligible therapeutic effect. This emotional connection to a treatment that can occur has the potential to affect a patient's long-term wellbeing.

In order to support wellbeing, it may be necessary for post-trial care for a long-term optogenetic trial to include access to a therapist or counsellor to support any adjustments to circumstances. There is a moral argument to provide support in order to help participants transition on from a trial. From a research perspective, this will also produce useful information on how people respond to living with optogenetic implants. This is information that can be beneficial to the neuroscience community as neural implants become more common. The varying emotional responses to brain implants are not fully understood, and yet with the potential for non-therapeutic use in the future making the use of neural implants more widespread, understanding fully the range of responses to living with a brain implant becomes more important.

Together, these elements can result in a significant additional burden to sponsors and researchers. But the provision of post-trial access and care can be made easier by planning and costing at the very start to ensure provision and to help relieve the burden on research staff. For smaller organisations, it may be appropriate for additional insurance to be obtained if the necessary funding is not in place at the start of a trial. Clinical trials are required to have insurance to cover public and product liability as well as no fault compensation. This is in case of adverse reactions to an investigational treatment or harm caused by negligence. An extension to clinical trial insurance to cover ongoing support for patients receiving benefit when a study closes early will minimise the financial risk to sponsors while also providing participants with reassurance and support.

\section{The Proposed Path Forward}

Much attention has been paid to ensuring that patients are fully engaged and supported when they are approached about taking part in research. But ensuring that the same level of attention is provided at the end of research is equally as important. Post-trial support and care for participants is vital for any research and a world-wide standardized approach to that care should be developed. At the start of this paper four conditions were identified by sponsors as being necessary for the provision of post-trial access. The conditions that optogenetics is likely to be considered for will predominantly be serious and chronic as the long-term and invasive nature of optogenetic therapy would otherwise result in an inappropriate risk profile. As optogenetics is a treatment and not a cure, the withdrawal or switching off of an implant can also be 
expected to result in the resumption of symptoms. The invasive nature of optogenetics will also result in the treatment predominantly being used after the use and failure of other alternative treatments. Using a sponsor's own list of conditions for post-trial access of experimental treatments, providing there is a positive risk/benefit profile, there will therefore be a strong argument for sponsors to provide some degree of ongoing access to an optogenetic treatment. With the imminent arrival of optogenetic therapies within the brain we recommend the following:

- Trials should be planned to allow life-long access, follow up and data collection for optogenetic therapies to the benefit of participants and science.

- Additional support should be factored in to support participant wellbeing as they transition between different trial phases.

- Devices should be designed and built as much as possible with future proofing in mind.

- Both therapy efficacy and patient wellbeing should be considered when considering post-trial care.

- Funders and sponsors need to cost in at the start for long-term patient support, for example by obtaining additional insurance.

Through a little bit of forward planning, sponsors can ensure that this is all done without significant disruption. Oversight by ethical and regulatory bodies can ensure that clinical trials are not approved if sponsors do not ensure that these issues are satisfactorily addressed in applications. While this review has focused on the use of optogenetics within the brain many of the issues apply to other tissue types, highlighting the importance of standardized guidance and regulations. For if these long-term implications are not addressed, then sponsors may find that people may be less willing to take part in such intrusive research, the impact of which will be felt much wider than the research community.

\section{Acknowledgments}

This work was supported by the CANDO project (http://www.cando.ac.uk/), a collaborative project to develop an optogenetic therapy for the treatment of focal epilepsy. CANDO is funded through Wellcome (grant number 102037) and Engineering and Physical Sciences Research Council (grant number NS/A000026/1) through an Innovative Engineering for Health grant.

\section{Disclosure}

The authors report no conflicts of interest in this work.

\section{References}

1. World Medical Association. World Medical Association Declaration of Helsinki: ethical principles for medical research involving human subjects. JAMA. 2013;310(20):2191-2194. doi:10.1001/jama.2013.281053

2. Council for International Organizations of Medical Sciences in collaboration with the World Health Organization. International ethical guidelines for health-related research involving humans; 2016. Available from: https://cioms.ch/wp-content/uploads/2017/01/WEB-CIOMS-EthicalGuidelines. pdf. Accessed May 21, 2021.

3. European Union. Regulation (EU) 2017/745 of the European Parliament and of the Council of 5 April 2017 on medical devices, amending Directive 2001/83/EC, Regulation (EC) No 178/2002 and Regulation (EC) No 1223/2009 and repealing Council Directives 90/385/EEC and 93/42/ EEC. Available from: https://eur-lex.europa.eu/legal-content/EN/TXT/PDF/?uri=CELEX:32017R0745. Accessed May 21, 2021.

4. European Union. Regulation (EU) 1394/2007 of the European Parliament and of the Council of 13 November 2007 on advanced therapy medicinal products and amending Directive 2001/83/EC and Regulation (EC) No 726/2004. Available from: https://eur-lex.europa.eu/LexUriServ/ LexUriServ.do?uri=OJ:L:2007:324:0121:0137:en:PDF. Accessed May 21, 2021.

5. Aldinger C, Bierer B, Li R, et al. MRCT center post-trial responsibilities framework continued access to investigational medicines. guidance document. Version 1.2; 2017. Available from: https://mrctcenter.org/wp-content/uploads/2017/12/2017-12-07-Post-Trial-Responsibilities-GuidanceDocument-Version-1.2.pdf. Accessed May 20, 2021.

6. Centre for Research on Multinational Corporations (SOMO). Post-trial access to treatment. Corporate best practices; 2015. Available from: https:// www.somo.nl/wp-content/uploads/2015/02/Post-trial-access-to-treatment.pdf. Accessed May 18, 2021.

7. Guru A, Post RJ, Ho YY, Warden MR. Making sense of optogenetics. Int J Neuropsychopharmacol. 2015;18(11):pyv079. doi:10.1093/ijnp/pyv079

8. Keshmiri Neghab H, Soheilifar MH, Grusch M, et al. The state of the art of biomedical applications of optogenetics. Lasers Surg Med. 2021. doi:10.1002/1sm.23463

9. Liu S, Tang Y, Xing Y, Kramer P, Bellinger L, Tao F. Potential application of optogenetic stimulation in the treatment of pain and migraine headache: a perspective from animal studies. Brain Sci. 2019;9(2):26. doi:10.3390/brainsci9020026

10. Wykes RC, Kullmann DM, Pavlov I, Magloire V. Optogenetic approaches to treat epilepsy. J Neurosci Methods. 2016;260:215-220. doi:10.1016/j. jneumeth.2015.06.004 
11. Wang KW, Ye XL, Huang T, Yang XF, Zou LY. Optogenetics-induced activation of glutamate receptors improves memory function in mice with Alzheimer's disease. Neural Regen Res. 2019;14(12):2147-2155. doi:10.4103/1673-5374.262593

12. Gensight. Dose-escalation study to evaluate the safety and tolerability of GS030 in Subjects with Retinitis Pigmentosa (PIONEER). NLM identifier: NCT03326336. Available from: http://www.clinicaltrials.gov/ct2/show/NCT03326336. Accessed December 1, 2021.

13. Allergan. RST-001 phase I/II trial for advanced retinitis pigmentosa. NLM identifier: NCT02556736. Available from: http://clinicaltrials.gov/ct2/ show/NCT02556736. Accessed December 1, 2021.

14. Nanoscope Therapeutics Inc. Efficacy and safety of vMCO-010 optogenetic therapy in adults with retinitis pigmentosa (RESTORE). NLM identifier: NCT04945772. Available from: https://clinicaltrials.gov/ct2/show/NCT04945772. Accessed December 1, 2021.

15. Nanoscope Therapeutics Inc. Dose-escalation study to evaluate the safety and tolerability of intravitreal vMCO-I in patients with advanced retinitis pigmentosa. NLM identifier: NCT04919473. Available from: https://clinicaltrials.gov/ct2/show/NCT04919473. Accessed December 1, 2021.

16. Sahel JA, Boulanger-Scemama E, Pagot C, et al. Partial recovery of visual function in a blind patient after optogenetic therapy. Nat Med. 2021;27:1223-1229. doi:10.1038/s41591-021-01351-4

17. Nanoscope therapeutics. Bedford, Texas: Press Release; 2021. Available from: https://nanostherapeutics.com/2021/06/03/nanoscopes-optogeneticgene-therapy-restores-clinically-meaningful-vision/. Accessed November 30, 2021.

18. Rapeaux AB, Constandinou TG. Implantable brain machine interfaces: first-in-human studies, technology challenges and trends. Curr Opin Biotechnol. 2021;72:102-111. doi:10.1016/j.copbio.2021.10.001

19. Wheless JW, Gienapp AJ, Ryvlin P. Vagus nerve stimulation (VNS) therapy update. Epilepsy Behav. 2018;88:2-10. doi:10.1016/j. yebeh.2018.06.032

20. Sadelain M. Insertional oncogenesis in gene therapy: how much of a risk? Gene Ther. 2004;11(7):569-573. doi:10.1038/sj.gt.3302243

21. Zhang D, Chen Q, Shi C, et al. Dealing with the foreign-body response to implanted biomaterials: strategies and applications of new materials. Adv Funct Mater. 2021;31(6):2007226. doi:10.1002/adfm.202007226

22. Shen Y, Campbell RE, Côté DC, Paquet ME. Challenges for therapeutic applications of opsin-based optogenetic tools in humans. Front Neural Circuits. 2020;14:41. doi:10.3389/fncir.2020.00041

23. Delbeke J, Haesler S, Prodanov D. Failure modes of implanted neural interfaces. In: Guo L, editor. Neural Interface Engineering: Linking the Physical World and the Nervous System. Cham: Springer; 2020:123-172.

24. European Medicines Agency. Guideline on follow-up of patients administered with gene therapy medicinal products (EMEA/CHMP/GTWP/60436/ 2007). Available from: https://www.ema.europa.eu/en/documents/scientific-guideline/guideline-follow-patients-administered-gene-therapy-medic inal-products_en.pdf. Accessed May 25, 2021.

25. European Medicines Agency. Guideline on safety and efficacy follow-up - risk management of advanced therapy medicinal products (EMEA/ 149995/2008). Available from: https://www.ema.europa.eu/en/documents/scientific-guideline/guideline-safety-efficacy-follow-risk-managementadvanced-therapy-medicinal-products en.pdf. Accessed May 26, 2021.

26. Drew L. "Like taking away a part of myself"-life after a neural implant trial. Nat Med. 2020;26(8):1154-1156. doi:10.1038/d41591-020-00028-8

27. Palmerini E. A legal perspective on body implants for therapy and enhancement. Int Rev Law Comp Technol. 2015;29(2-3):226-244. doi:10.1080/ 13600869.2015 .1055664

28. Fins JJ. Deep brain stimulation, deontology and duty: the moral obligation of non-abandonment at the neural interface. $J$ Neural Eng. 2009;6 (5):050201. doi:10.1088/1741-2552/6/5/050201

29. Underwood E. Researchers grapple with the ethics of testing brain implants. Science. 2017. doi:10.1126/science.aar3698

30. Rossi PJ, Giordano J, Okun MS. The problem of funding off-label deep brain stimulation: bait-and-switch tactics and the need for policy reform. JAMA Neurol. 2017;74(1):9-10. doi:10.1001/jamaneurol.2016.2530

31. Lázaro-Muñoz G, Yoshor D, Beauchamp MS, Goodman WK, McGuire AL. Continued access to investigational brain implants. Nat Rev Neurosci. 2018;19(6):317-318. doi:10.1038/s41583-018-0004-5

32. Sierra-Mercado D, Zuk P, Beauchamp MS, et al. Device removal following brain implant research. Neuron. 2019;103(5):759-761. doi:10.1016/j. neuron.2019.08.024

33. Cho HL, Danis M, Grady C. Post-trial responsibilities beyond post-trial access. Lancet. 2018;391(10129):1478-1479. doi:10.1016/S0140-6736(18) 30761-X

34. Hendriks S, Grady C, Ramos KM, et al. Ethical challenges of risk, informed consent, and posttrial responsibilities in human research with neural devices: a review. JAMA Neurol. 2019;76(12):1506-1514. doi:10.1001/jamaneurol.2019.3523

35. Lawton J, Blackburn M, Rankin D, et al. Broadening the debate about post-trial access to medical interventions: a qualitative study of participant experiences at the end of a trial investigating a medical device to support type 1 diabetes self-management. AJOB Empirical Bioethics. 2019;10 (2):100-112. doi:10.1080/23294515.2019.1592264

36. Saver RS. At the end of the clinical trial: does access to investigational technology end as well. $W N$ Eng $L$ Rev. 2009;31:411.

37. Richardson HS, Belsky L. The ancillary-care responsibilities of medical researchers. An ethical framework for thinking about the clinical care that researchers owe their subjects. Hastings Cent Rep. 2004;34:25-33. doi:10.2307/3528248

38. Gilbert F, Goddard E, Viaña JNM, Carter A, Horne M. I miss being me: phenomenological effects of deep brain stimulation. AJOB Neurosci. 2017;8(2):96-109. doi:10.1080/21507740.2017.1320319

39. Leuenberger M, Klein E, Specker Sullivan L. Losing meaning: philosophical reflections on neural interventions and their influence on narrative identity. Neuroethics. 2021;1-15. doi:10.1007/s12152-021-09468-6

40. Gilbert F, Viaña JNM, Ineichen C. Deflating the "DBS causes personality changes" bubble. Neuroethics. 2018;14:1-17. 
Open Access Journal of Clinical Trials

Dovepress

\section{Publish your work in this journal}

The Open Access Journal of Clinical Trials is an international, peer-reviewed, open access journal publishing original research, reports, editorials, reviews and commentaries on all aspects of clinical trial design, management, legal, ethical and regulatory issues, case record form design, data collection, quality assurance and data auditing methodologies. The manuscript management system is completely online and includes a very quick and fair peer-review system, which is all easy to use. Visit http://www.dovepress.com/testimonials.php to read real quotes from published authors.

Submit your manuscript here: https://www.dovepress.com/open-access-journal-of-clinical-trials-journal 